\title{
La lectura y el futuro profesor: Una aproximación a sus creencias acerca de la lectura*
}

\author{
Reading and the future teacher: \\ An approach to their beliefs about reading
}
Carolina González Ramírez ${ }^{* *}$, Marcelo González Figueroa ${ }^{* * *}$, Constanza Lobos Guerrero ${ }^{* * * * *}$, Jorge Valenzuela ${ }^{* * * * *}$, Carla Muñoz

\section{RESUMEN}

Con el objetivo de indagar en las creencias de futuros docentes Palabras clave: acerca de la lectura, se realizó un análisis de contenido textual de tipo mixto. Participaron 364 docentes en formación de Educación Básica $(n=202)$ y Educación Parvularia $(n=162)$ provenientes de nueve universidades chilenas. Los discursos de estos futuros docentes dan cuenta de creencias diversas referentes creencias, formación de lectores, formación de profesores, lectura. a la lectura (inter e intrasujetos) que van desde la herramienta hasta la práctica cultural. Contra lo esperado, se constata empíricamente un predominio de nociones caracterizadas por un bajo número de facetas y por un énfasis en definiciones desde lo experiencial, independientemente de la carrera y de los años de formación. Así, la clasificación de creencias, considerando de manera simultánea la riqueza y la especialización, muestra una alta concentración en el tipo experiencial simple, es decir, con

Esta investigación ha sido financiada por la Comisión Nacional de Investigación en Ciencia y Tecnología (Conicyt-Chile) a través del Proyecto Fondecyt Regular 1170779.

** Chilena. Doctora en Didáctica de la Lengua y la Literatura. Profesora Asociada, Pontificia Universidad Católica de Valparaíso, Valparaíso, Chile. carolina.gonzalez.r@ pucv.cl

**** Chileno. Sociólogo. Consultor. Universidad de Chile, Santiago, Chile. marcelogonzalezfigueroa@gmail.com

***** Chilena. Magíster en Sociología @ C. Universidad Alberto Hurtado, Santiago, Chile. coni.lobosguerrero@gmail.com

****** Chileno. Doctor en Ciencias de la Educación. Académico, Universidad Católica del Maule, Talca, Chile.jvalenzuela@ucm.cl

****** Chilena. Doctora en Ciencias Psicológicas y de la Educación. Académico, Universidad Católica del Maule, Talca, Chile.cmunozv@ucm.cl (Autor correspondiente) 
baja especialización y baja riqueza (71\%), mientras que el tipo especializada compleja (alta especialización y riqueza) solamente alcanza un $11 \%$. Junto con discutir las implicancias de estos hallazgos para la práctica pedagógica y la formación de profesores lectores, se aporta adicionalmente un esquema de análisis para las creencias en torno a la lectura que puede contribuir a nuevos estudios relacionados con este tópico.

\begin{abstract}
To investigate the beliefs of future teachers about reading, we conducted a mixed-type textual content analysis. 364 future teachers of Primary Education $(n=202)$ and Early Childhood Education $(n=162)$ from 9 Chilean universities participated in this study. These future teachers reveal diverse beliefs about reading (inter and intrasubject) that range from tools to cultural practice. Contrary to expectations, we verified a predominance of notions characterized by a low number of facets and an emphasis on definitions from the experiential, independent of the career, and years of training. Thus, the classification of beliefs, simultaneously considering wealth and specialization, shows a high concentration in the simple experiential type, with low specialization and low richness (71\%).

In contrast, the complex, specialized type (high specialization and richness) only reaches $11 \%$. Besides discussing the complex implications of these findings for pedagogical practice and reading teachers' training, we also provide an analysis scheme for reading beliefs. This could contribute to new studies on this subject.
\end{abstract}




\section{Introducción}

Progresivamente el estudio del profesor como lector ha adquirido relevancia en la investigación educativa; esto porque se considera que los hábitos lectores de los docentes tendrían incidencia no solo en su formación personal, sino también a nivel profesional (Granado y Puig, 2015; Rudland \& Kemp, 2004) y de manera específica, en la forma en que enfrentarán la enseñanza de la lectura y la formación de nuevos lectores ${ }^{1}$ comprometidos en el contexto escolar. En efecto, esta línea de estudios asume la hipótesis de que un profesor con un hábito lector consolidado será probablemente un mejor mediador en el proceso lector.

Los estudios ya clásicos de Applegate y colaboradores $(2004 ; 2014)$ han alertado respecto de la necesidad de considerar las instancias de disfrute con la lectura y de goce estético como parte de la formación inicial de profesores. Estos espacios serían necesarios para modelar formas de acercamiento al proceso, procurando instancias de apreciación estética a las que probablemente no han tenido acceso previamente. En estas instancias los futuros profesores desarrollarían formas de acercamiento a lo escrito donde reaccionan de manera profunda, crítica y comprometida ante cualquier tipo de texto. Como consecuencia de esto, sería posible producir una resignificación y revalorización de sus propias prácticas lectoras. En este sentido, estudios recientes como el de Gaete-Moscoso (2019) muestran cómo los docentes establecen comparaciones entre sus representaciones en torno a la lectura y el discurso establecido ("narrativa social"). Según la autora, estas comparaciones pueden llevarlos a invisibilizar sus propias lecturas por considerarlas poco valiosas, lo que les impediría revelarse como lectores en la interacción con sus estudiantes. Ello explicaría prácticas descontextualizadas de lectura, sin anclaje en las experiencias personales y en donde primaría un objetivo evaluativo.

Respecto de la actividad pedagógica, estudios referidos a la cognición docente reconocen que esta se encuentra informada por sabe-

La expresión "formación de lectores" es utilizada en este contexto en el sentido de "formación de lectores comprometidos (motivados) con la lectura". Esta concepción no reniega de una etapa necesaria en el desarrollo lector, esto es, el aprendizaje y dominio del código, pero reconoce que aunque necesaria, no es suficiente para asegurar el desarrollo de un hábito lector. 
res, creencias y pensamientos que interactúan entre sí. El caso de la lectura y la formación de nuevos lectores no sería una excepción. En este sentido, las nociones de "sujet lecteur didactique" (Delbrayelle \& Duszynski, 2007) o la de "sujet lecteur enseignant" (Émery-Bruneau, 2011), cristalizan esta idea acerca del educador como lector y mediador, asumiendo la idea de una relación entre las prácticas personales de lectura del profesor - en este caso, literarias y su autoimagen lectora-, lo que tendría un impacto en la forma en que media la lectura en el espacio escolar. Asimismo, las creencias acerca de qué es la lectura, su naturaleza y cómo se enseña y se aprende pueden determinar el margen de acción docente en el aula. Si como suponemos, los profesores sostienen un sistema complejo de creencias en torno a los objetos de enseñanza, la lectura —uno de los objetos que más se identifican con el proceso educativo - no estaría exenta de estas creencias.

En el caso de profesores de enseñanza básica y educadoras de párvulo se hace necesaria la exploración de sus creencias en torno a la lectura por dos razones fundamentales. En primer término, se trata de profesionales cuyo rol fundamental es iniciar a niñas y niños formalmente en la cultura escrita. En segundo lugar, la formación inicial docente se centra en saberes disciplinares y didácticos, asumiendo la cualidad de lectores competentes y con hábito lector consolidado de estos futuros docentes (Ministerio de Educación de Chile, Mineduc, 2014; 2018c). Sin embargo, la evidencia hasta ahora disponible no apoya esta premisa y más bien llama a tener cautela respecto de este dato (Deleuze, 2009; Dengler, 2018; Granado y Puig, 2014; Nathanson, Pruslow \& Levitt, 2008). Por ello, conocer las creencias de los docentes en cuanto al acto de leer y la lectura nos lleva a un doble propósito. Por una parte, establecer una "línea de base" desde la cual comenzar su formación, incluyendo la lectura como una clave de conocimiento personal y, por otra, como un elemento de construcción identitaria (Granado y Puig, 2015), para configurar un "profesor-lector" o sujeto lector didáctico.

\subsection{La lectura como objeto multidimensional}

La lectura puede ser definida como acto, proceso e incluso, como producto. En tales definiciones, se actualiza al menos una faceta de este constructo, lo que deja entrever la complejidad de una definición consensuada. Lo anterior se explica por el hecho de que la lectura es a la 
vez una herramienta mental y una práctica social que es resignificada por las comunidades (Chartier y Hébrard, 2000; Ferreiro, 2001; Manguel, 1997). En este sentido, cabe considerar que la lectura comprende tanto procesos lingüísticos, psicológicos, así como también prácticas socioculturales (Kalman, 2017).

Desde una perspectiva cognitiva, la lectura puede ser entendida como un proceso mental complejo e interactivo que requiere de habilidades psicolingüísticas para decodificar y comprender. Se asume que el lector es quien construye el significado e interpreta a partir de sus esquemas de conocimientos previos y de la nueva información presentada en el texto (Kintsch, 2018; McNamara, 2010; Richter, 2015).

Desde una perspectiva sociocultural, se entiende la lectura como una práctica que aunque posee una dimensión personal, adquiere sentido pleno cuando se comparte con otros, se dialoga, se discute y se lleva a cabo en comunidades, esto es, compartiendo esta herramienta cultural (Gee, 2015; Rogoff, Paradise, Arauz, Correa-Chávez \& Angelillo, 2003; Sénéchal \& LeFevre, 2014).

Asimismo, la lectura puede también ser definida a partir de las funciones que le son atribuidas, es por ello que hacemos mención a una dimensión utilitaria, pues en la actualidad se tiende a asociar la lectura principalmente con el aprendizaje y el estudio, lo que nos lleva a concebirla como actividad intelectual (Larrañaga y Yubero, 2005).

\subsection{Relación del profesor con la lectura}

Dada esta multiplicidad de sentidos, es posible pensar que para un profesor ciertas facetas de la lectura pudieran estar más presentes o más alineadas con sus creencias acerca de este objeto. Lo cierto es que la investigación en torno a la relación del profesor con la lectura ha mostrado que esta se ve influida por conocimientos (didácticos y disciplinares) a la vez que experienciales. Estas creencias serían una suerte de "epistemologías personales" con las cuales el profesor actuaría en su labor pedagógica.

\subsection{Creencias del futuro profesor acerca de la lectura}

La investigación al respecto tiene como supuesto que existe un conocimiento propio del docente que incide directamente en su intervención 
didáctica (Borg, 2015). Dicho pensamiento estaría constituido por los saberes y creencias que motivarían su acción pedagógica.

En cuanto a las creencias, si bien se han identificado características comunes a este constructo, no es menos cierto que consensuar una definición resulta complejo (Fives \& Buehl, 2012; Pajares, 1992). Conceptualmente, se trataría de construcciones mentales subjetivas formadas muy tempranamente en la vida del individuo y que están compuestas por actitudes y valores de la enseñanza, los estudiantes y el proceso educativo, los que operarían a nivel funcional, guiando al sujeto en la planificación de la conducta, la interpretación de eventos y en la realización de inferencias prácticas (Jiménez, Rodríguez, Suárez y O'Shanahan, 2014).

A nivel de estructura, las creencias se organizarían en sistemas que actuarían como filtros para comprender y dar sentido al mundo (Borg, 2015) y tenderían a perpetuarse incluso ante contradicciones provocadas por procesos de razonamiento, tiempo, escolarización o experiencia. Del mismo modo, los profesores adoptarían diferentes creencias educacionales dependiendo de variables tales como el género, los años de experiencia docente o la materia que enseñan (Suárez y Jiménez, 2014).

Aun cuando la investigación en torno a las creencias ha aumentado considerablemente en los últimos años, según Maggioni, Fox y Alexander (2015) los estudios específicos de creencias en relación con la lectura son aún limitados, aunque de muy diversa naturaleza. En efecto, una revisión de la literatura realizada por estas autoras muestra una diversidad de temas que van desde las creencias acerca de la naturaleza de la lectura y de qué significa ser lector, hasta las relaciones entre creencias y prácticas pedagógicas, factores que influencian las creencias de los docentes, creencias sobre la enseñanza de la lectura y cómo esta se desarrolla, entre otros.

En relación con estos estudios, vale la pena mencionar el de Shaw y Mahlios (2011), quienes exploraron las creencias de los futuros profesores de primaria a partir de las metáforas de alfabetización, en el contexto de un curso de dos semestres de enseñanza de la lectura. Después del curso, la mayoría de los profesores en formación mantuvieron sus creencias, reforzando la idea de cuán estables y resistentes al cambio pueden ser estas. 
Otros estudios revisados por Hall (2005) han abordado las creencias de los profesores respecto de la lectura en otras áreas de contenido, revelando que la mayoría de los docentes de secundaria parecían conceptualizar la lectura de manera simple, como un conjunto de habilidades básicas adquiridas en los primeros años de escolaridad. Coincidentemente con esto, los profesores no creían que sus estudiantes requirieran apoyo en la lectura en los grados superiores.

En cuanto a las creencias del profesor como lector, en el estudio de Tercanlioglu (2001) los profesores en formación creían de manera mayoritaria que un buen profesor debía necesariamente ser un buen lector que lee junto con sus alumnos. En tanto, el estudio de Gupta (2004) muestra dos perfiles de profesores en formación: aquellos cuya aproximación es "leer por placer" mientras que para otro grupo leer solo equivale a informarse. Asimismo, las estrategias de este último grupo se concentraban en "recordar", más que en comprender o evaluar la información. Esta dicotomía tendría serias consecuencias en la forma en la que estos profesionales abordarían la enseñanza en el futuro.

Dados estos antecedentes y siguiendo a Jiménez et al. (2015), un paso necesario para cualquier programa de desarrollo docente es conocer las creencias de los profesores para poder intervenir de manera eficaz y eficiente. En este contexto, profundizar en cómo los futuros profesores chilenos conciben la lectura podría darnos pistas para apoyar la formación inicial en la línea de configurar un "profesor-lector".

\section{Método}

Este estudio se inserta dentro de un proyecto mayor referido al profesor como lector, cuyo objetivo fue caracterizar las creencias de docentes en formación en torno a la lectura, así como la relación de estas lecturas con su formación personal y profesional.

El presente artículo indaga específicamente en las creencias que detentan los futuros profesores respecto de la lectura, por medio de la pregunta "Para mí, leer es...." Asumimos que luego de la respuesta a esta pregunta se actualizarían los diversos modos o facetas en que se concibe el objeto ("la lectura"). Así, si un sujeto solo concibe la lectura como una herramienta para el conocimiento, su definición debería relevar esa faceta, con prescindencia de otras (esto es, como un medio de 
entretención, un vínculo con otros, etc.). En la misma línea y respecto de los futuros profesores, la respuesta a esta pregunta podría darnos pistas acerca de qué facetas del objeto poseen preeminencia en su sistema de creencias y con ello, comprender cómo los futuros profesores mediarán este objeto cultural en la sala de clases.

Planteamos un análisis de contenido textual mixto cuyo objetivo era identificar, en el corpus discursivo de la pregunta "Para mí, leer es...", el nivel de prevalencia que poseen las diversas facetas de la lectura en los futuros profesores, considerando su nivel de riqueza y grado de especialización. El supuesto de esta opción analítica fue que las creencias podrían verse afectadas por la carrera (Educación Parvularia y Educación Básica) y por el año de estudio; esto por cuanto la formación explícita de lectura y su didáctica en pedagogía básica podría influir en esta diferenciación, tanto entre carreras como a nivel de avance curricular.

El cruce entre la riqueza (número de facetas presentes en la definición) y el grado de especialización disciplinar permite considerar cuatro casos teóricos en el análisis, que se presentan en la Figura 1 y se explican a continuación.

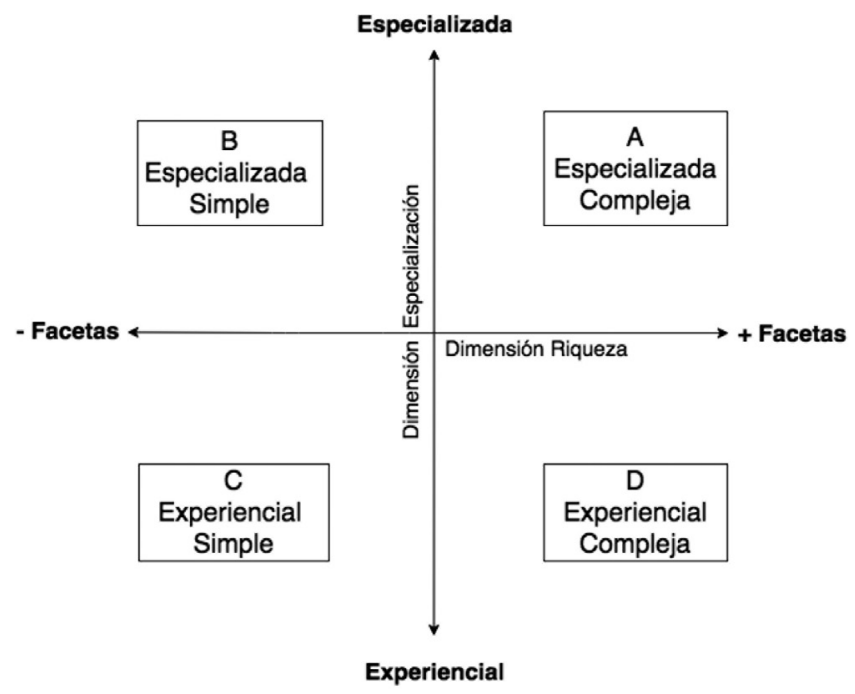

Figura 1. Tipos de definiciones de acuerdo con las categorías de especialización y riqueza.

Fuente: Elaboración propia 
- Aproximación experiencial-simple. Corresponde a las definiciones que esbozan entre cero a una faceta de la lectura y carecen de un nivel de especificidad, ya que el saber evidenciado corresponde a uno de tipo experiencial. Asimismo, suelen identificarse atribuciones positivas de la lectura a través de las que se releva su valor socialmente reconocido.

- Aproximación experiencial-compleja. En esta categoría se observan definiciones de lectura ancladas en la experiencia, relevando dos o tres facetas de la lectura.

- Aproximación especializada simple. En esta categoría, se proponen definiciones de lectura con un nivel mayor de especificidad, dando cuenta de un conocimiento declarativo más especializado y con utilización de conceptos propios del área de formación.

- Aproximación especializada compleja. Corresponde a una conceptualización de la lectura en la que se evidencia claramente un saber disciplinar especializado.

\subsection{Participantes}

El estudio contó con la participación de 364 estudiantes, futuros profesores de educación básica $(n=201)$ y educación de párvulos $(n=162)$, provenientes de nueve universidades chilenas. Se trató de alumnos que cursaban entre primero y quinto año de carrera. La muestra en estudiantes de educación de párvulos correspondió a un $100 \%$ de mujeres y en educación básica (primaria) a un $84,1 \%$ de mujeres, porcentaje similar a la media nacional para esta carrera (Servicio de Información de Educación Superior, SIES, 2019).

\subsection{Instrumentos}

La recolección de datos se realizó a partir de un cuestionario que indagaba en las prácticas lectoras y que combinaba preguntas abiertas y cerradas.

Para dar cuenta de las creencias que subyacen en torno a la lectura en esta población se consideró la respuesta a la pregunta "Para mí, leer es.... Formulada de esta manera, la interrogante permitía al sujeto relevar los aspectos más prominentes del objeto (lectura) y de su relación personal - en tanto futuro profesor-con la lectura. 
Adicionalmente, se registraron datos relativos a la carrera y a los años cursados como estudiantes de pedagogía, lo que permitió clasificarlos en etapa inicial ( $\left.1^{\circ} \mathrm{año}\right)$, intermedia $\left(2^{\circ}\right.$ y $3^{\circ}$ años $)$ y final $\left(4^{\circ}\right.$ y $5^{\circ}$ años) de la carrera, y con ello, establecer si existían diferencias cualitativas en sus creencias en función del avance en su trayecto formativo.

\subsection{Procedimiento}

El criterio único de inclusión en este estudio consistió en ser estudiantes en las carreras de Educación Parvularia o Pedagogía Básica; ello, bajo la premisa de que las creencias en torno a la lectura podrían variar en función de la formación y del año de la carrera. Para ello, fueron contactados varios programas de formación pedagógica a lo largo de Chile. Las instituciones que respondieron a este llamado fueron nuevamente contactadas para fijar un día y hora de presentación del proyecto a sus estudiantes en sus dependencias.

Antes de proceder a la aplicación del cuestionario, se siguió un estricto protocolo de presentación de la investigación a los participantes, donde se les explicaron los objetivos del estudio, se les aseguró que los datos serían confidenciales y que toda información recogida sería solo conocida por el equipo de investigación. Todos los estudiantes que accedieron a participar firmaron un documento de consentimiento previo a la aplicación. Al mismo tiempo, quienes no quisieron responder el instrumento tuvieron la libertad de no hacerlo. La aplicación del cuestionario fue realizada de manera presencial, mediante un formulario impreso. Como retribución por su participación, al final de la aplicación los estudiantes recibieron un pequeño incentivo no monetario. Los datos recolectados fueron posteriormente digitados en una base de datos, lo que implicó la codificación numérica en el caso de las preguntas cerradas y la transcripción textual y literal de las respuestas a las preguntas abiertas.

\subsection{Procedimientos analíticos}

\subsubsection{Desarrollo y evaluación de un libro de códigos}

Para el desarrollo y evaluación de un sistema de códigos, que es el eje del análisis de información realizado, utilizamos como referencia el sistema de libro de códigos grupal propuesto por MacQueen, McLe- 
llan, Kay y Milstein (1998). Este sistema se basa en la elaboración progresiva de un sistema de códigos que facilita el trabajo de codificación en equipo. Implica esencialmente elaborar una herramienta que logra claridad sobre el sentido y el uso que debe hacerse de los códigos que se utilizarán para organizar la información textual. Es un método que está especialmente pensado en el trabajo de equipos de codificación de volúmenes relevantes de información obtenidos a partir de entrevistas y sintetiza estrategias que son utilizadas indistintamente para análisis cualitativos y cuantitativos. El sistema propuesto por este equipo consideró especificar para cada código una etiqueta o nombre breve, una definición compartida y ejemplos de cuándo usar o no cada código. La versión utilizada consideró los elementos esenciales y utilizó los ejemplos "de cuándo no utilizar el código" solo en aquellos casos en que la complejidad del caso lo ameritaba (ver Anexo I).

Una de las ventajas de utilizar este sistema de codificación es que permite contar con un corpus textual organizado según un sistema de clasificación con valor teórico, así como también posibilita elaborar consultas simples de la frecuencia de ciertos códigos en el corpus (análisis cuantitativo) y además, permite realizar consultas de parcelas del corpus para realizar interpretaciones fundadas en los datos y controlar la interpretación cuando se analiza la diversidad de formas expresivas que contiene una categoría o código de interés (análisis cualitativo). Este estudio utilizó ambos procedimientos de manera complementaria.

Para llevar a cabo este proceso se desarrolló una codificación que integró una estrategia deductiva e inductiva. En un primer momento, se establecieron categorías teóricas que pudieran ayudar a comprender la información, mientras que en el transcurso de la codificación se añadieron aquellos nodos emergentes de los discursos de los futuros profesores que no fueron contemplados en el comienzo.

El libro de códigos elaborado en la investigación sintetizó tres elementos:

- Definiciones iniciales orientadas teóricamente.

- Aprendizajes realizados por la lectura del corpus y el análisis exploratorio de datos.

- Discusiones grupales del equipo de investigación. 


\subsubsection{Libro de códigos definitivo}

A fin de establecer un sistema de codificación confiable, se utilizaron dos estrategias de verificación. En un primer momento, se dividió el corpus en partes iguales de manera que dos codificadores categorizaran a partir de la matriz de códigos establecidos. Una vez realizada la tarea, se procedió a hacer la revisión cruzada de los casos. En esta etapa, logramos un acuerdo de un $78 \%$. En aquellos casos en que no hubo acuerdo entre codificadores se ajustó el sistema de códigos y sus definiciones, para poder mejorar el análisis cualitativo de la pregunta. Finalmente, se ajustó el libro de códigos según los acuerdos suscritos, lográndose un acuerdo del $100 \%$ entre los codificadores.

En un segundo momento, la confiabilidad en la codificación fue evaluada mediante acuerdo interjueces, que buscó que la codificación fuera recreable en circunstancias distintas o con codificadores distintos (Krippendorff, 1997). La codificación fue realizada por dos investigadoras independientes, miembros del equipo, quienes codificaron 37 casos seleccionados al azar. Para esta tarea se utilizó el software Nvivo 12-Plus (QSR, 2018). Esta codificación arrojó un índice de consistencia del 98\%, el cual implica un índice Kappa de $82 \%$ (Shan \& Wang, 2017).

\subsubsection{Codificación de la totalidad del corpus}

Una vez elaborado el sistema de codificación y evaluados los resultados preliminares en cuanto a su consistencia, procedimos a codificar la totalidad del corpus. Los resultados que presentamos a continuación consideran de manera complementaria las estadísticas descriptivas de cada uno de los códigos y análisis que dan cuenta de la diversidad expresiva que implica el material textual clasificado.

\subsubsection{Dimensiones de análisis}

A partir del tratamiento y codificación de los datos consignados en el corpus de definiciones, se levantaron categorías de análisis con el propósito de caracterizar las definiciones. En primera instancia, consideramos la riqueza y en segundo lugar, la especificidad (especialización) de la definición. A continuación, presentamos la conceptualización de las dimensiones señaladas. 


\subsubsection{Riqueza}

Esta dimensión corresponde a la cantidad de facetas de la lectura que los sujetos elicitan en su discurso. Así, se estableció que la riqueza puede ser simple o compleja de acuerdo con el número de facetas identificadas en cada verbalización. De este modo, en la riqueza simple la cantidad de facetas elicitadas iba de 0 a 1 , mientras que en la riqueza compleja la cantidad de facetas elicitadas iba de 2 a 3 . Cabe señalar que se determinaron tres facetas de la lectura a partir de las dimensiones presentadas en el marco teórico: faceta cognitiva, faceta social y faceta funcional.

La faceta cognitiva abarca todas aquellas respuestas que hacen referencia a la lectura como un proceso cognitivo complejo; esto, porque en ella estarían involucrados distintos niveles de procesamiento de información. En esta faceta se contemplan todas las creencias que conciben la lectura como una habilidad lingüística, por ejemplo, lectura limitada a decodificación y también aquellas que refieren a la comprensión, entendida como proceso cognitivo de orden superior (Kintsch, 2018). Por su parte, la faceta social como lo indica su nombre, enfatiza el rol social de la actividad de lectura. Se trata de algo que sucede no solo en la cabeza de las personas (como es concebido desde la psicología cognitiva), sino que más bien corresponde a prácticas que involucran a comunidades que comparten formas de lectura y significados culturales en torno al acto de leer (Gee, 2015). Por tanto, esta faceta de la lectura contiene aquellas referencias a la lectura como práctica social, ya que se entiende que la lectura se realiza en comunidades o espacios en donde los sujetos comparten herramientas culturales. Finalmente, la faceta funcional contiene aquellas creencias que refieren a la lectura como un medio que posibilita otras situaciones o como un objeto que les permite realizar acciones diversas. Se distingue de la precedente porque solo es considerada en términos individuales. Dentro de esta categoría es posible distinguir dos tipos de funciones: la función fruitiva, que corresponde a aquellas definiciones que identifican la lectura como un medio de disfrute, mientras que la función pragmática dice relación con las concepciones en donde la lectura permite alcanzar ciertos propósitos, dependiendo del lector, el contexto y el tipo de texto/género al que se enfrente. 


\subsubsection{Especialización}

Esta dimensión refiere al origen preeminente del conocimiento que subyace en las verbalizaciones de lectura analizadas. Como creencias en torno a la lectura, estas podrían estar constituidas por experiencias personales con el objeto (en tanto lector) como por conocimiento especializado, en grados diversos. Lo experiencial remite al saber derivado de las experiencias personales en torno a la lectura, lo que se revelaba en el discurso por medio de referencias asociadas al disfrute personal, la búsqueda de evasión, de conocimiento y desarrollo personal. En tanto, lo especializado refiere a creencias acerca de la actividad lectora ancladas en conocimiento técnico especializado. Se identifica por la utilización de terminología técnica (por ejemplo, decodificación, comprensión y habilidad). Esta referencia a saberes disciplinares podría explicarse por la socialización académica que han tenido los futuros profesores en su proceso de formación inicial, lo que se justifica porque para hacer uso de dicha terminología los futuros docentes deben haber pasado por un proceso de enseñanza formal en el ámbito disciplinar.

\section{Resultados}

\subsection{Riqueza de la noción de lectura}

El análisis de las respuestas a la pregunta "Para mí, leer es..." consideró tanto su riqueza (cantidad de facetas elicitadas en la respuesta) como su caracterización de manera más específica en cuanto a las facetas de la lectura aludidas en las respuestas (cognitiva, social y funcional).

La gran mayoría de las respuestas de nuestros participantes aludió a una sola dimensión de la lectura, denotando una importancia prevalente o una mirada restringida del objeto. En concreto, solo uno de cada cinco de estos futuros profesores hizo referencia a más de una faceta al describir la lectura (ver Tabla 1).

Del total de respuestas, los sujetos elicitaron 416 facetas de la lectura, las que fueron clasificadas en tres categorías principales: facetas cognitiva, social y funcional.

En términos globales, los resultados evidenciaron un predominio de la faceta funcional (71\%), seguida de la faceta cognitiva (25\%) y de la faceta social con solo un $4 \%$ de menciones. 
Tabla 1.

Distribución de definiciones según categoría riqueza.

\begin{tabular}{ccc}
\hline Categoría riqueza & $\begin{array}{c}\text { Riqueza simple } \\
(0-1)\end{array}$ & $\begin{array}{c}\text { Riqueza compleja } \\
(2-3)\end{array}$ \\
\hline Respuestas & 289 & 72 \\
\hline Porcentaje frecuencia & $80,1 \%$ & $19,9 \%$ \\
\hline
\end{tabular}

Fuente: Elaboración propia.

La comparación por carreras muestra una tendencia similar (Figura 2). La faceta funcional de la lectura es predominante en las menciones de estudiantes de Pedagogía Básica y Educación Parvularia, seguida por las menciones de la faceta cognitiva. En cambio, la faceta social de la lectura está presente en menor medida en las definiciones de ambos grupos.

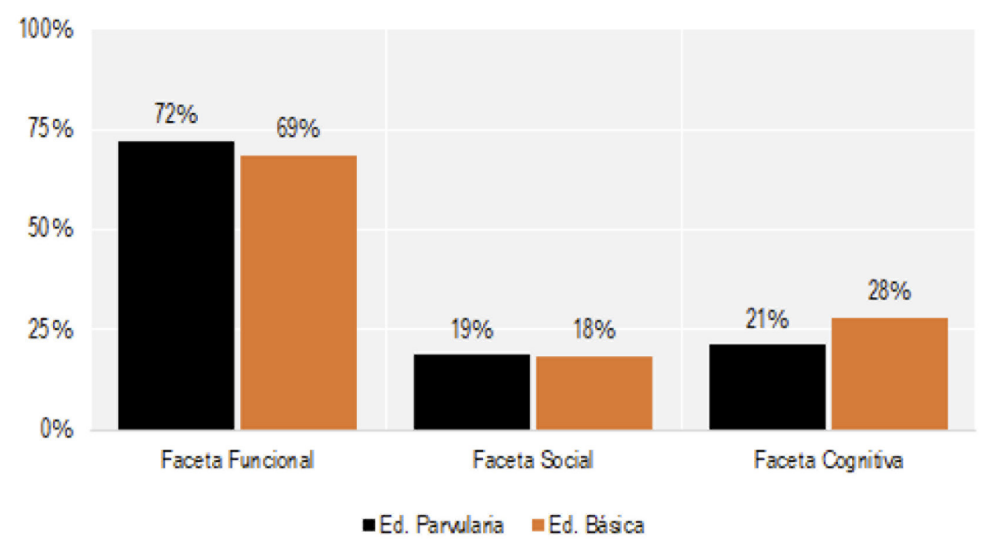

Figura 2. Porcentaje de facetas mencionadas de la lectura, según carrera. Fuente: Elaboración propia.

A partir de esta primera clasificación fue posible identificar creencias específicas en cada una de estas facetas ${ }^{2}$.

En la faceta funcional se identificaron cuatro ideas fundamentales:

La referencia a los participantes se realizó por medio de una letra y un código numérico $(\mathrm{P}=$ participante + número). 
- Valor social de la lectura. Este se expresa en términos de importancia, sin mayores especificaciones de lenguaje disciplinar: "importante ya que es un factor primordial en la educación y en la vida" (P45); "importante y fundamental para el desarrollo de la humanidad" (P94).

- La lectura es un medio de acceso al conocimiento. Es decir, se concibe la lectura como una herramienta que permite acceder a la información y facilitaría el "aprender, tener la posibilidad de abrir nuevas puertas de conocimiento. El leer me lleva a informarme, aprender y entretenerme" (P51).

- La lectura permite desarrollar la imaginación. Los informantes tienden a utilizar la metáfora del viaje para dar cuenta de que a través de los textos pueden conocer otras realidades y transportarse a otros mundos, porque es "una forma de viajar a través de la imaginación" (P163).

- El hábito lector depende del grado de motivación. Para los informantes, es patente la idea de creer que si se siente gusto por la tarea, habría un mayor compromiso con esta y por tanto, se realiza la acción de mejor manera. Así, el acto de leer se define como "entretenido cuando me gusta el libro, a veces aburrido cuando no me gusta lo que estoy leyendo" (P131).

En el caso de la faceta cognitiva también identificamos creencias que sintetizamos en tres ideas fundamentales:

- La lectura es la capacidad de decodificar un texto. Esto alude a una concepción netamente lingüística de la lectura donde leer es "la capacidad de decodificar signos lingüísticos, y a la vez poner parte de mi experiencia cuando se hace esta codificación" (P13).

- La lectura es un proceso cognitivo complejo, a través del cual el sujeto construye significados y comprende. Más allá de la decodificación, leer es para los participantes del estudio "un proceso de comprensión y análisis de un discurso textual estableciendo relaciones de ideas y conceptos para construir un significado" (P96).

Finalmente, cabe hacer mención a la faceta social, que tiene un porcentaje de frecuencia menor respecto de las anteriores. Las creencias subyacentes remiten, principalmente, a la lectura como una prác- 
tica que implica una interacción social. De esta manera, para algunos de los informantes leer excede la dimensión individual e incorpora lo social como elemento estructurante de la actividad: "proceso no natural que implica la decodificación. Práctica social, cultural e histórica. Acto interpretativo" (P18).

\subsection{Especialización de la noción de lectura}

Un segundo aspecto evaluado en este estudio es el grado de especialización de las respuestas. Tal como se aprecia en la Tabla 2, la especialización conceptual no es un rasgo propio de las definiciones analizadas. Tres de cada cuatro de estos futuros mediadores de la lectura $(76,2 \%)$ elaboraron sus respuestas acerca de qué es leer a partir de su experiencia individual, sin incorporar elementos especializados que hayan sido parte de su formación profesional específica. El bajo porcentaje de aparición del conocimiento especializado correspondiente a 23,8\% podría ser un indicador de que existe cierta precariedad conceptual en la formación inicial recibida por ambos grupos de informantes, ya que el uso de lenguaje disciplinar es reducido.

Tabla 2.

Porcentajes de aparición y frecuencia bruta de tipo de especialización del conocimiento.

\begin{tabular}{lll}
\hline Categoría especialización & Experiencial & Especializado \\
\hline Porcentaje de aparición & $76,2 \%$ & $23,8 \%$ \\
\hline Frecuencia bruta & 275 & 86 \\
\hline
\end{tabular}

Fuente: Elaboración propia.

\subsection{Interrelaciones entre riqueza y grado de especialización}

Según lo señalado en nuestro supuesto, esperábamos que las creencias de los estudiantes de pedagogía pudieran verse afectadas por la carrera que cursaban (Educación de Párvulos y Pedagogía Básica) y el año de estudio. Esto, considerando que la formación explícita en torno a la lectura y su didáctica en el caso de Pedagogía Básica podría influir en esta diferenciación, tanto entre carreras como a nivel de avance curricular.

El cruce entre la riqueza (número de facetas presentes en la definición) y el grado de especialización nos permitió identificar los cuatro tipos elaborados teóricamente y dimensionar su presencia en nuestra 
muestra. En la Figura 3 se presenta la configuración de estos grupos asociados al cruce de las dos dimensiones.

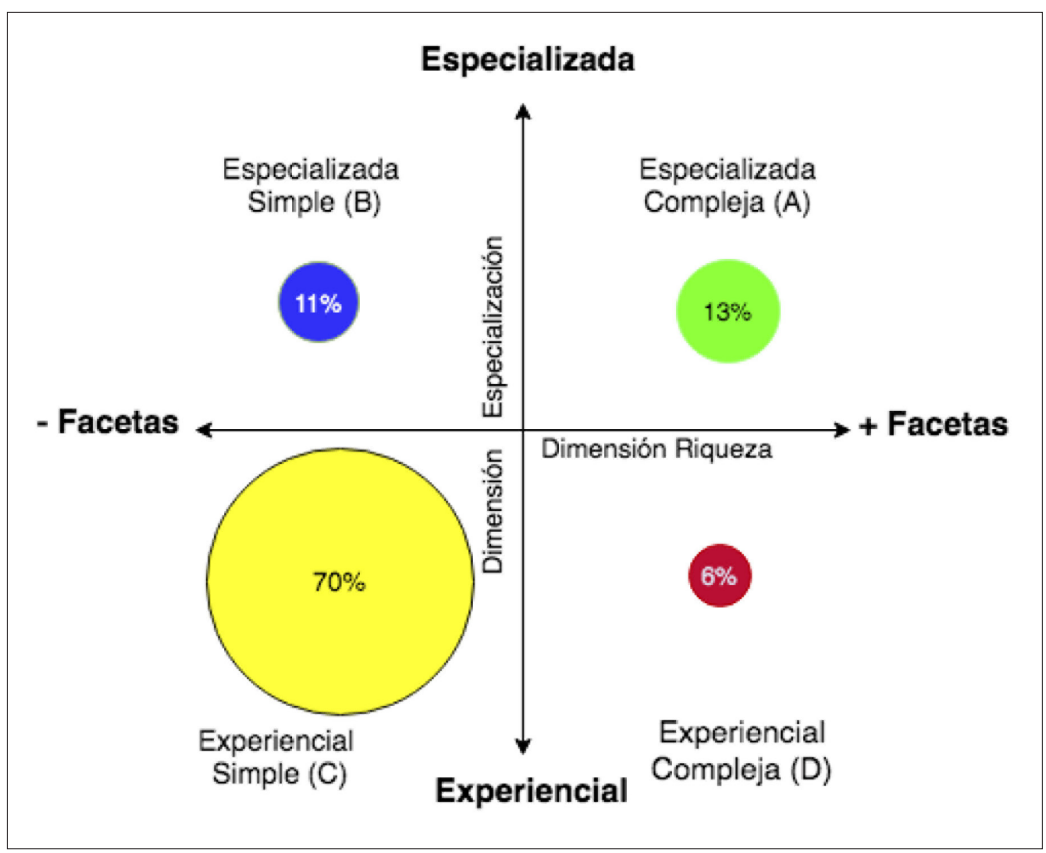

Figura 3. Resultados por tipos de aproximación nocional de lectura según grado de riqueza (cantidad de facetas) y especialización.

Fuente: Elaboración propia.

\subsubsection{Definición especializada compleja}

Corresponde a una conceptualización de la lectura en la que se evidencia claramente un saber disciplinar especializado. En este tipo de definición es posible identificar dos a tres facetas del objeto, lo que implica el reconocimiento de la complejidad del constructo. Ahora bien, del total de definiciones del corpus analizado, 48 fueron susceptibles de ser categorizadas como especializadas-complejas, es decir, un $13 \%$ del total. El mismo porcentaje se repitió para el análisis por carrera, de modo que no existirían mayores diferencias según disciplina. Sin embargo, resulta interesante observar la distribución de este tipo de definiciones, según la etapa de la carrera que cursan los estudiantes. Precisamente, y en concordancia con la hipótesis que guio esta investigación, el $63 \%$ de las definiciones de este tipo son desarrolladas 
por estudiantes que se encuentran en la etapa final de sus estudios de pedagogía. De este modo, es posible avanzar en la hipótesis de que la socialización y adquisición de conocimientos de carácter disciplinar generarían una modificación de las creencias en torno a la lectura, lo que se explicita en sus definiciones. Ahora bien, si se compara entre carreras, son los estudiantes de etapa final de Educación Básica quienes presentan la mayor cantidad de definiciones de este tipo (29\% contra $21 \%$ de Educación Parvularia).

\subsubsection{Definición especializada simple}

Las menciones a facetas de la lectura son limitadas (0-1). A partir de los análisis, tenemos que este tipo de definiciones corresponde a un $11 \%$ del total que conforman el corpus, es decir, 38 casos. Mientras que del total de participantes de la carrera de Educación Parvularia 11\% elicitan definiciones de este tipo, para el caso de Educación Básica esta cifra corresponde a un $\mathbf{1 0 \%}$, lo que denota diferencias mínimas entre ambas disciplinas. Si se analiza por etapa de la carrera que cursan, las definiciones de este tipo se concentran en la etapa intermedia para ambas carreras (16\% para Educación Parvularia y 18\% para Educación Básica), lo que sería coherente con la progresión curricular de los estudiantes, pues se requiere conocimiento técnico para construir una definición.

\subsubsection{Definición experiencial-simple}

Corresponde a aquellas definiciones que aluden solo a una faceta de la lectura y carece de un nivel de especificidad técnico, utilizando un vocabulario que está más cercano al uso cotidiano que al tecnolecto. Los análisis arrojaron la presencia de 251 definiciones del corpus categorizadas como experiencial-simple, lo que corresponde a un $70 \%$ del total del corpus de definiciones recogidas. El análisis por carreras muestra que el $71 \%$ de los estudiantes de Educación Parvularia y el 68\% de los estudiantes de Educación Básica presenta este tipo de definiciones.

Un análisis similar según la etapa de formación (inicial, intermedia y final) muestra que la mayor cantidad de definiciones experiencialessimples se concentra en la etapa inicial, para ambas carreras (54\%). Estos datos sostendrían la hipótesis de una relación entre la riqueza de sus definiciones y el nivel de formación especializada alcanzado. 


\subsubsection{Definición experiencial-compleja}

Del total de definiciones construidas por los participantes, tan solo el $6 \%$ desarrolló definiciones que se inscriben en este grupo, siendo la categoría menos presente en el corpus. Si vemos la distribución en cada carrera, solo un 4,4\% de la muestra de estudiantes de Educación Parvularia presentó definiciones de este tipo, mientras que de Educación Básica esta cifra llegó a 8,4\%. En relación con las etapas de la carrera que cursan los participantes, la mayoría de las definiciones de este tipo fue elicitada por estudiantes en su etapa inicial (69\%).

\section{Discusión y conclusiones}

El objetivo de este estudio fue identificar las creencias de futuros docentes en torno a la lectura, bajo la premisa de que la riqueza de sus discursos en torno a la pregunta "Para mí, leer es..." daría cuenta de creencias más o menos sofisticadas que permitirían o impedirían procesos efectivos de mediación escolar de la lectura. En nuestra muestra, observamos que la mayor cantidad de respuestas pudieron identificarse con el tipo experiencial simple y que las referencias mayoritariamente aluden a aspectos funcionales de la lectura.

El análisis realizado muestra, por una parte, una tensión entre la experiencia personal y el mandato profesional de formar nuevos lectores. A nivel profesional, se observa efectivamente una tendencia a concebir la lectura de manera más especializada (utilizando, por ejemplo, terminología técnica), conforme se avanza en el trayecto de formación. Ello podría implicar una influencia de los procesos de formación pedagógica en sus saberes disciplinares. ¿Implica ello que las creencias de estos futuros profesores hayan cambiado durante la formación? Esta es una pregunta que queda abierta y que requeriría de un estudio longitudinal.

Si bien la movilidad de creencias es un tema aún en debate en la comunidad científica, es posible advertir al mismo tiempo una fuerte tendencia a identificar la obligatoriedad de la lectura como una amenaza. El gusto por leer está asociado con lo voluntario, por lo que cuando ello es demandado externamente, es visto como una imposición y condición suficiente para el desagrado o el abierto rechazo. Esto es interesante desde el punto de vista formativo, pues se trata de futuros educadores que también son expuestos a lecturas obligatorias en su 
formación profesional, las que en sus discursos aparecen como elementos que atentan contra su tiempo de lectura personal. Esta asociación placer/libertad generaría una tensión evidente en su ethos pedagógico, según el cual el profesor debe procurar experiencia de goce con la lectura para motivar al futuro lector (Mineduc, 2018a; 2018b). Estos datos refuerzan los hallazgos de estudios precedentes (Lobos, Muñoz y Valenzuela, 2020).

En segundo término y asociada al punto anterior, la concepción utilitaria de la lectura es la más referida por los profesores en formación. De este modo, las creencias más recurrentes dan cuenta de que la lectura es entendida como una herramienta que permite acceder al conocimiento, así como también posibilita el desarrollo de la imaginación, además de presentar beneficios intrínsecos, manifestados en el valor socialmente reconocido de la lectura. Ahora bien, el predominio de la faceta funcional habla también de una relación con la lectura marcada por la utilidad. Esta dimensión aparece como prevalente en estudiantes universitarios y ha sido reportada en estudios sobre lectura académica (Muñoz, Valenzuela, Avendaño y Nuñez, 2016). Ello muestra que para el futuro profesor, la utilidad es un elemento indiscutible de la lectura, lo que sumado al discurso socialmente aprendido respecto de los beneficios intrínsecos de esta práctica lo llevará a seguir insistiendo con ella en sus actuaciones pedagógicas. En este sentido, estudios recientes como el de Munita y Pérez (2013), muestran que la concepción de los profesores acerca de la lectura y su rol en el currículo determinan el margen de acción en el aula, influyendo incluso en el tipo de prácticas evaluativas, con lo cual se empobrecen las interacciones con las diferentes facetas de este objeto cultural.

En la misma línea, observamos que las creencias en estos futuros docentes respecto de la lectura se basan en experiencias muy concretas y discretas, asociadas esencialmente con el plano individual y/o formativo. En efecto, la mención a la lectura se realiza en espacios formales (ámbito escolar y universitario), sin que haya existido mención a la lectura como práctica social, vinculada con el tiempo libre u ocio. Esta situación se observa en los dos grupos de futuros educadores, lo que también merece una consideración especial, puesto que no observamos diferencias entre futuras educadoras de párvulo y profesores de enseñanza básica. En este sentido, interpela la ausencia de referencias 
a prácticas de lectura compartida en el caso de educadoras de párvulo, lo que según la investigación potencia el desarrollo del lenguaje oral y posteriormente el acceso a lo escrito.

No obstante ello, observamos que la carrera y los años de formación incidirían en la transformación de sus creencias, ya que estas se van complejizando al adquirir saberes teóricos que dan mayor sustento a sus creencias. Es así como se observó que los estudiantes de años superiores son quienes elaboran definiciones más complejas y especializadas, dando cuenta de que sus creencias comienzan a superar los límites de la experiencia. Esto último da pie a pensar que intervenciones focalizadas durante la formación, centradas en enriquecer el corpus de lecturas personales de los futuros profesores, así como el ejercicio de prácticas lectoras colegiadas, tenderían a enriquecer las creencias del futuro profesor y potenciarían en él una identidad lectora fuerte.

Al respecto, el ejemplo de experiencias con clubes docentes de lectura (Commeyras, Bisplinghoff \& Olson, 2003; Cremin, Mottram, Collins, Powell \& Safford, 2009) muestra que este tipo de intervenciones puede provocar cambios en la identidad lectora del docente, enriqueciéndola y posicionándose como sujeto lector-enseñante. Sostenemos que, para mejorar los procesos de formación de nuevos lectores en la escuela, es necesario propiciar un modelo de formación inicial tendiente a enriquecer la figura del sujeto lector didáctico, ya que de estos futuros mediadores depende la calidad de la relación que nuestros niños y niñas establecerán con este objeto cultural complejo.

\section{Referencias bibliográficas}

Applegate, A. J. \& Applegate, M. D. (2004). The Peter effect: Reading habits and attitudes of preservice teachers. The Reading Teacher, 57(6), 554-563. http://doi.org/10.1080/ 19388071.2014.898719

Applegate, A. J., Applegate, M. D., Mercantini, M. A., McGeehan, C. M., Cobb, J. B., DeBoy, J. R., Modla, V. B., \& Lewinski, K. E. (2014). The Peter effect revisited: Reading habits and attitudes of college students. Literacy Research and Instruction, 53(3), 188204. https://doi.org/10.1080/19388071.2014 .898719 
Borg, S. (2015). Teacher cognition and language education: Research and practice. London: Bloomsbury Publishing.

Cassany, D. (2006). Tras las líneas. Sobre la lectura contemporánea. Barcelona: Anagrama.

Chartier, A.-M. y Hébrard, J. (2000). Saber leer y escribir: unas "herramientas mentales" que tienen su historia. Infancia y Aprendizaje, 89(1), 11-24.

Commeyras, M., Bisplinghoff, B. S., \& Olson, J. (Eds.). (2003). Teachers as readers: Perspectives on the importance of reading in teachers' classrooms and lives. Newark: International Reading Association.

Cremin, T., Mottram, M., Collins, F., Powell, S., \& Safford, K. (2009). Teachers as readers: Building communities of readers. Literacy, 43(1), 11-19. https://doi.org/10.1111/j.17414369.2009.00515.x

Delbrayelle, A. \& Duszynski, M. (2007). De la difficulté à se construire comme un sujet lecteur didactique quand on est professeur des écoles stagiaire. En M. Lebrun, A. Rouxel, \& C. Vargas (Eds.), La littérature et l'école. Enjeux, résistances, perspectives (pp. 101-111). Provence: Presses Universitaires de Provence.

Deleuze, G. (2009). Futurs instituteurs et faibles lecteurs: réalité et/ou fatalité. Enjeux, 74, 93-100.

Dengler, K. A. (2018). Alliterate pre-service teachers' reading histories: An exploratory multiple case study. Graduate Dissertations and Theses, 44. https://orb.binghamton.edu/dissertation_ and_theses $/ 44$

Émery-Bruneau, J. (2011). Former des "sujets-lecteurs-enseignants": une responsabilité collective pour des actions didactiques réfléchies. Québec français, 163, 79-81. https://id.erudit.org/ iderudit/65428ac

Ferreiro, E. (2001). Pasado y presente de los verbos leer y escribir. México, D.F.: Fondo de Cultura Económica.

Fives, H. \& Buehl, M. M. (2012). Spring cleaning for the "messy" construct of teachers' beliefs: What are they? Which have been examined? What can they tell us? En K. R. Harris, S. Graham, T. Urdan, S. Graham, J. M. Royer, \& M. Zeidner (Eds.), APA educational psychology handbook, vol. 2. Individual differences and cultural and contextual factors (pp. 471-499). https://doi.org/10.1037/13274-019 
Gaete-Moscoso, R. C. (2019). Escenas de lectura: ¿qué repertorio usan los docentes que enseñan a leer para hablar de su práctica lectora? Literatura y Lingüística, 40, 209-227. http://dx.doi. org/10.29344/0717621x.40.2067

Gee, J. P. (2015). The new literacy studies. En J. Rowsell \& K. Pahl (Eds.), The Routledge Handbook of literacy studies. London: Routledge. https://doi.org/10.4324/9781315717647.ch2

Granado, C. y Puig, M. (2014). ¿Qué leen los futuros maestros y maestras? Un estudio del docente como sujeto lector a través de los títulos de libros que evocan. Ocnos: Revista de Estudios sobre Lectura, 11, 93-112. https://doi. org/10.18239/ocnos_2014.11.05

Granado, C. y Puig, M. (2015). La identidad lectora de los maestros en formación como componente de su identidad docente. Un estudio de sus autobiografías como lectores. Ocnos: Revista de Estudios sobre Lectura, 13, 43-63. https:// doi. org/10.18239/ocnos_2015.13.03

Gupta, R. (2004). Old habits die hard: Literacy practices of pre-service tea- chers. Journal of Education for Teaching, 30(1), 67-78. https://doi.org/10.1080/0260747032000162325

Hall, L. A. (2005). Teachers and content area reading: Attitudes, beliefs and change. Teaching and Teacher Education, 21(4), 403414. https://doi.org/10.1016/j.tate.2005.01.009

Jiménez, J. E., Rodríguez, C., Suárez, N., y O’Shanahan, I. (2014). ¿Coinciden nuestras ideas con lo que dicen las teorías científicas sobre el aprendizaje de la lectura? Revista Española de Pedagogía, 72(259), 397-414. http://www.jstor.org/stable/24726629

Jiménez, J. E., Rodríguez, C., Suárez, N., O'Shanahan, I., Villadiego, Y., Uribe, C., Villalobos, J. A., \& Rodas, P. (2015). Teachers' implicit theories of learning to read: A cross-cultural study in Ibero-American countries. Reading and Writing, 28(9), 13551379. https://doi.org/10.1007/s11145-015-9574-z

Kalman, J. (2017). Literacies in Latin America. En B. V. Street \& S. May (Eds.), Literacies and Language Education (pp. 399-416). New York: Springer International Publishing. https://doi. org/10.1007/978-3-319-02252-9_30

Kintsch, W. (2018). Revisiting the construction-integration model of text comprehension and its implications for instruction. 
En D. E. Alvermann, N. J. Unrau, M. Sailors, \& R. B. Ruddell (Eds.), Theoretical models and processes of literacy (pp. 194219). London: Routledge.

Krippendorff, K. (1997). Metodología de análisis de contenido: Teoría y práctica. Buenos Aires: Paidos.

Larrañaga, E. y Yubero, S. (2005). El hábito lector como actitud. El origen de la categoría de "falsos lectores". Ocnos: Revista de Estudios sobre Lectura, 1, 43-60. https://doi.org/10.18239/ ocnos_2005.01.04

Lobos, C., Muñoz, C., y Valenzuela, J. (2020). Explorando las causas del descenso en la curiosidad intelectual durante la formación docente en Chile. REMIE Multidisciplinary Journal of Educational Research, 10(1), 25-46. https://dx.doi.org/10.17583/ remie.2020.4898

MacQueen, K. M., McLellan, E., Kay, K., \& Milstein, B. (1998). Codebook development for team-based qualitative analysis. Cultural Anthropology Methods, 10(2), 31-36. https://doi.org/1 $0.1177 / 1525822 X 980100020301$

Maggioni, L., Fox, E., \& Alexander, P. A. (2015). Beliefs about reading, text, and learning from text. En H. Fives \& M. Gregoire (Eds.), International handbook of research on teacher's belief (pp. 353-369). London: Routledge.

Manguel, A. (1997). A history of reading. New York: Penguin.

McNamara, D. S. (2010). Strategies to read and learn: Overcoming learning by consumption. Medical Education, 44(4), 340- 346. https://doi.org/10.1111/j.1365- 2923.2009.03550.x

Ministerio de Educación de Chile, Mineduc. (2014). Estándares orientadores para carreras de educación parvularia. Estándares pedagógicos y disciplinarios. Recuperado de https://bibliotecadigital.mineduc.cl/handle/20.500.12365/2235

Ministerio de Educación de Chile, Mineduc. (2018a). Bases Curriculares de la Educación Parvularia. Recuperado de https://parvularia.mineduc.cl/wp-content/uploads/sites/34/2018/03/ Bases_Curriculares_Ed_Parvularia_2018.pdf

Ministerio de Educación de Chile, Mineduc. (2018b). Bases curriculares. Primero a Sexto Básico. Recuperado de https://www.curriculumnacional.cl/614/articles-22394_bases.pdf 
Ministerio de Educación de Chile, Mineduc. (2018c). Estándares orientadores para egresados de carreras de pedagogía en educación básica. Recuperado de http://portales.mineduc.cl/ usuarios/cpeip/File/librosestandaresvale/libroparvulariafinal.pdf

Munita, F. y Pérez, M. (2013). "Controlar" las lecturas literarias: un estudio de casos sobre la evaluación en el plan de lectura complementaria de educación básica. Estudios Pedagógicos, 39(1), 179-198. https://doi.org/http://10.4067/S071807052013000100011

Muñoz, C., Valenzuela, J., Avendaño, C., y Núñez, C. (2016). Mejora en la motivación por la lectura académica: la mirada de estudiantes motivados. Ocnos, Revista de Estudios sobre Lectura, 15, 52-68. http://dx.doi.org/10.18239/oc- nos_2016.15.1.941

Nathanson, S., Pruslow, J., \& Levitt, R. (2008). The reading habits and literacy attitudes of in-service and prospective teachers: Results of a Questionnaire survey. Journal of Teacher Education, 59(4), 313-321. https://doi.org/10.1177/0022487108321685

Pajares, F. (1992). Teacher's beliefs and educational research: Cleaning up a messy construct. Review of Educational Research, 62(3), 307-332. https://doi.org/10.2307/1170741

QSR International (2018). Nvivo 12-Plus. [Software]. Recuperado de https://qsrinternational.com/nvivo

Richter, T. (2015). Validation and Comprehension of text information: Two sides of the same coin. Discourse Processes, 52(5-6), 337-355. https://doi.org/10.1080/016385 3X.2015.1025665

Rogoff, B., Paradise, R., Arauz, R. M., Correa-Chávez, M., \& Angelillo, C. (2003). Firsthand learning through intent participation. Annual Review of Psychology, 54(1), 175-203. https://doi. org/10.1146/annurev.psych.54.101601.145118

Rudland, N. \& Kemp, C. (2004). The professional reading habits of teachers: Implications for student learning. Australasian Journal of Special Education, 28(1), 4-17. https://doi. org/10.1080/1030011040280102

Sénéchal, M. \& LeFevre, J. A. (2014). Continuity and change in the home literacy environment as predictors of growth in vocabulary and reading. Child development, 85(4), 1552-1568. https://doi.org/10.1111/cdev.12222 
Shan, G. \& Wang, W. (2017). Exact one-sided confidence limits for Cohen's kappa as a measurement of agreement. Statistical Methods in Medical Research, 26(2), 615-632. https://doi. org/10.1177/0962280214552881

Shaw, D. M.\& Mahlios, M. (2011). Literacy metaphors of pre-service teachers: Do they change after instruction? Which metaphors are stable? How do they connect to theories? Journal of Education for Teaching, 37(1), 77-92. https://doi.org/10.1 080/02607476.2011.538274

Servicio de Información de Educación Superior, SIES. (2019). Bases de datos de matriculados en Educación superior 2019. Recuperado de https://www.mifuturo.cl/sies/

Suárez, N. y Jiménez, J. E. (2014). ¿Influyen los años de experiencia y la especialidad de los profesores en las teorías implícitas que se atribuyen sobre el aprendizaje de la lectura? Revista INFAD de Psicología, 2(1), 257-262. https://doi.org/http://dx.doi. org/10.17060/ijodaep.2014.n1.v2.438

Tercanlioglu, L. (2001). Pre-service teachers as readers and future teachers of EFL reading. TESL-EJ, 5(3), n3. https://doi.org/ https://www.tesl-ej.org/ej19/a2.html 


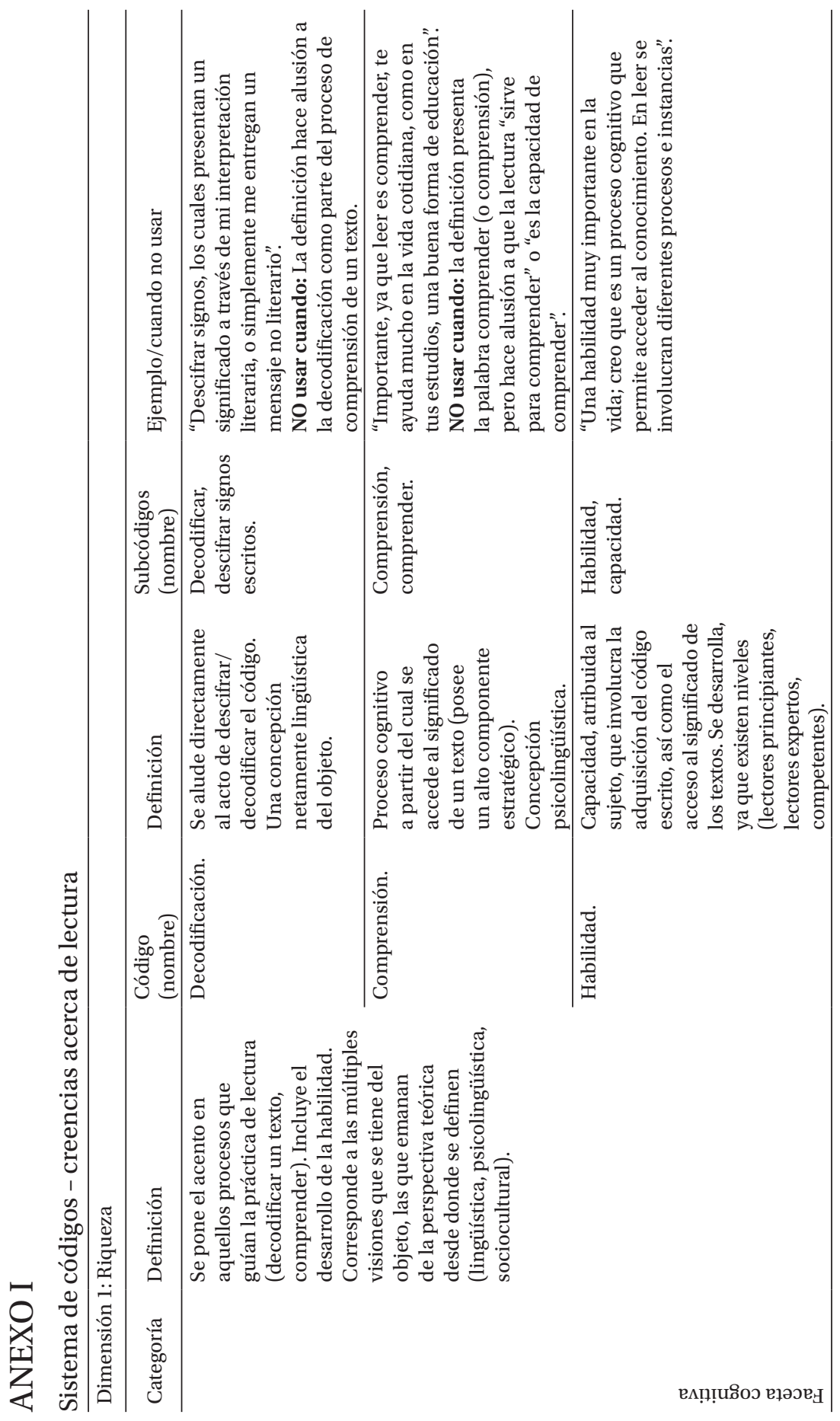




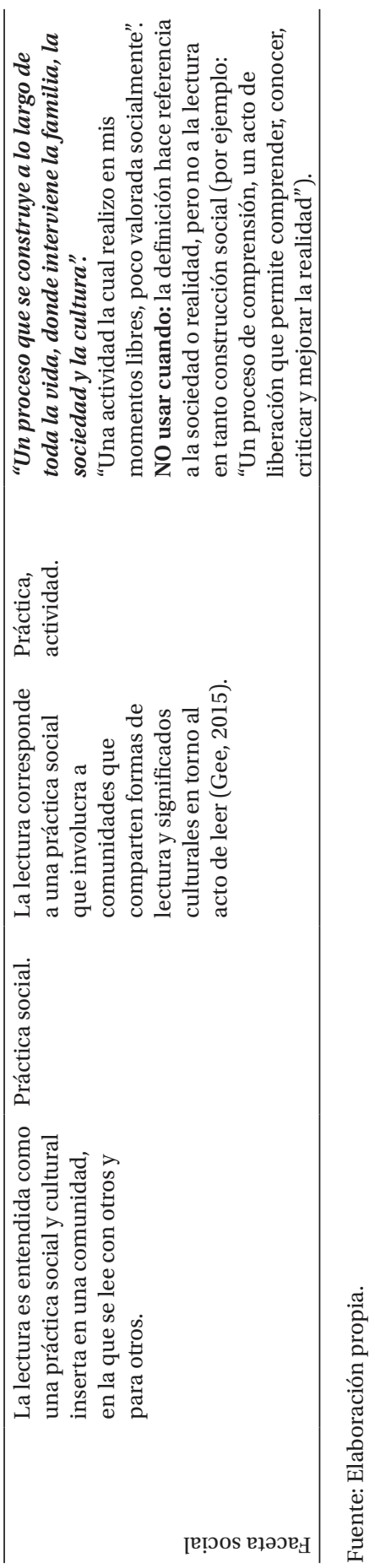




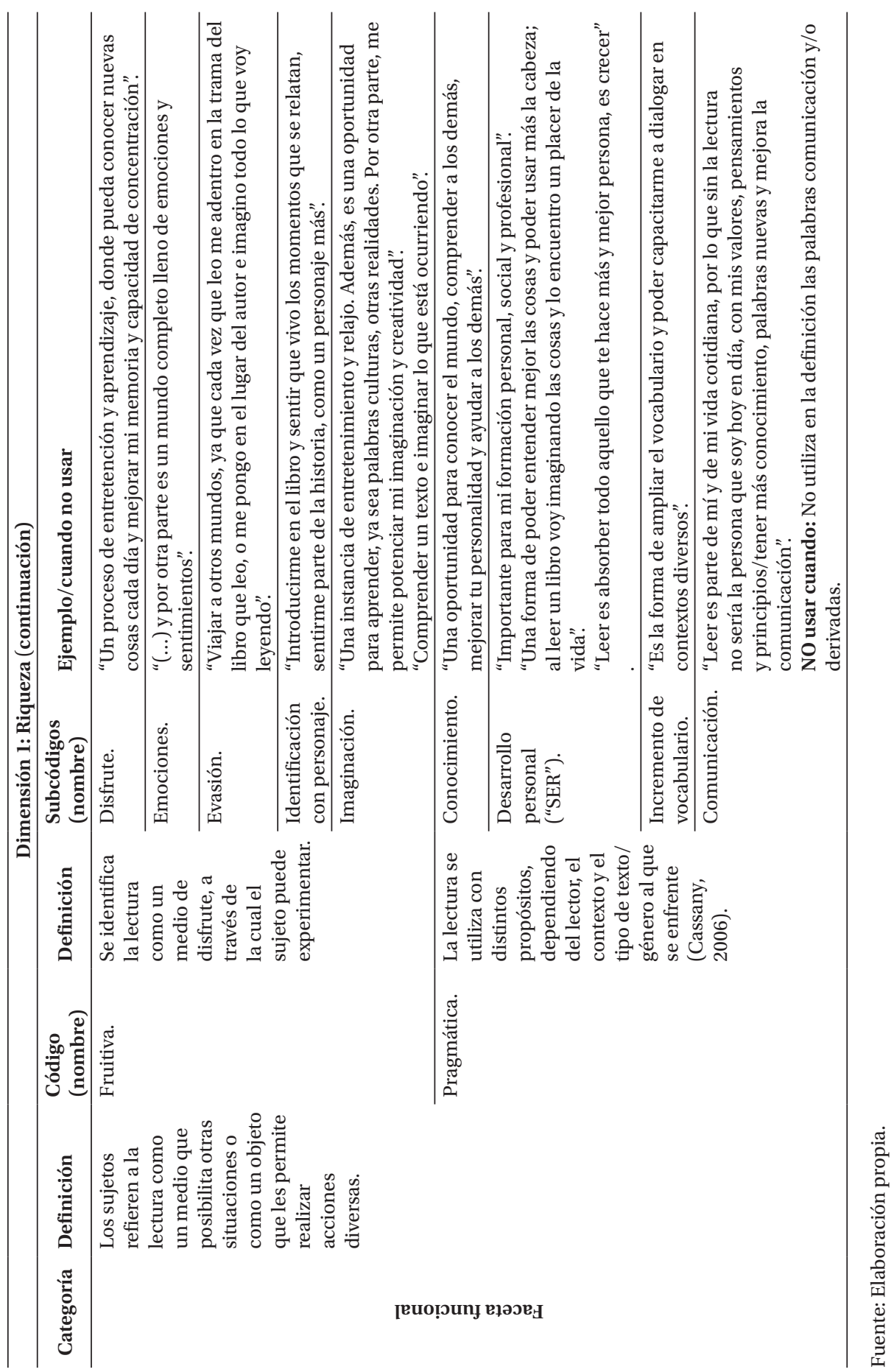




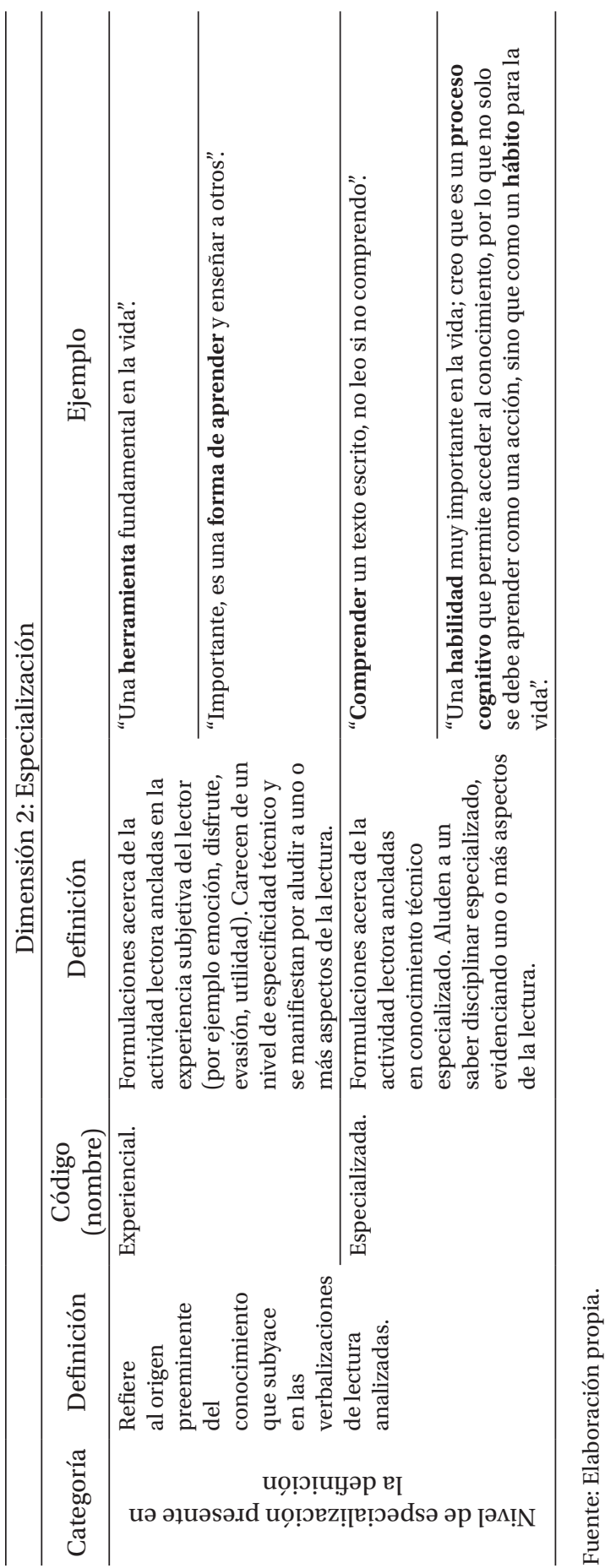

